\title{
Flow Investigation inside A Curved Square Duct
}

\author{
${ }^{1}$ Dipyaman Gangopadhyay, ${ }^{1}$ Niraj Kr. Prasad, ${ }^{1}$ A.K. Biswas, ${ }^{2}$ P.K. Sinha \\ ${ }^{I}$ National Institute of Technology, Durgapur, West Bengal, India \\ ${ }^{2}$ Durgapur Institute of Advanced Technology\& Management, West Bengal, India
}

\begin{abstract}
This paper presents the results of an experimental work with measurement of wall static pressure of $90^{\circ} \mathrm{C}$ shaped Curved duct. The test duct is made up of transparent perspex sheets to facilitate the flow visualization study. The duct has an inlet to exit area ratio of 1.0 with centerline distance of $750 \mathrm{~mm}$. The inlet aspect ratio of the test duct has been fixed at 1.0. The velocities for the proposed investigations are to be measured by using a Pitot tube.Wall pressures are measured with the help of an inclinedmanometer with the inclination of $35^{\circ}$. The manometer had two tubes emanating from it: one left open to the atmosphere and the other connected to the steel pipes attached to the four walls of the curved duct. The difference in the readings helped us calculate the static pressure and thereby the normalized pressure. Wall pressure distribution along the curved and parallel walls of the duct at $0^{\circ}, 22.5^{\circ}, 45^{\circ}, 67.5^{\circ}$ and $90^{\circ}$ measuring sections was measured. All the experimental data has been processed by an Intel i3 CPU, 3 GB RAM PC and analyzed to give the distribution of static pressure in the square duct.The main purpose of this investigation is to show the development of secondary flow which happens when the flow takes place through the bend in the curvature. This secondary flow arises as a result of a centrifugal force acting when the flow moves through the bend. The investigation is carried out at three different velocities $20 \mathrm{~m} / \mathrm{s}, 40 \mathrm{~m} / \mathrm{s}$ and $60 \mathrm{~m} / \mathrm{s}$. The distribution of normalized pressure which is the ratio of static pressure to the dynamic pressure is mapped and shown in the form of contours by using the software package SURFER.The trend of wall static pressure development on the walls of $\mathrm{C}$ shaped duct shows that as the flow proceeds towards the curvature, there exists a high pressure gradient between the outside face and inside face due the centrifugal force acting along the curvature. This shows the bulk shifting of flow towards the inside face. This is due to the generation of secondary motion in a plane perpendicular to the primary flow.

Keywords: C shapedcurved duct, Wall pressure,High pressure gradient, Secondary motion,SURFER
\end{abstract}

\section{INTRODUCTION}

Duct is a very old fluid mechanical device. Study of flow characteristics through a constant area duct is the foundation and fundamental research area of basic fluid mechanics since the concept of potential flow and frictional losses during the time of flow were established. Duct is nothing but a passage way made of sheet metal or other suitable material used for conveying air or other gas and liquid at different pressure. Planning, sizing, optimizing, detailing of duct work and also finding the pressure losses through a duct system is generally termed as duct design. The different types of ducts have been classified as straight, curved, annular, sector, C-shaped, S-shaped, Snake and Coil section, etc. Among all these ducts the common ones are straight, curved, and annular and sector ducts, which are used in most industrial applications.A large and important class of engineering problems is represented by internal viscous flows in curved duct. Accurate and reliable calculations of such flows are of great practical interest in the prediction of flow through inlets, nozzles, diffusers and other components of turbo machinery. The flow through a curved tube has attracted considerable attention not only because of its practical importance in chemical and mechanical engineering, but also because of physically interesting features under the action of centrifugal force caused by curvature of the tube. Internal flow through a duct is essential for obtaining improved performance of the overall propulsion system. In addition to the above, aircraft intakes, combustors, internal cooling system of gas turbines, ventilation ducts, wind tunnels etc. are the main application area of such ducts.Gao et. al. [1] in 2004 had investigated the effects of inclination angle of ribs on the flow behavior in rectangular ducts. The effect was investigated by Particle Image Velocimetry (PIV). The flow field description was based on the PIV results in planes both parallel and perpendicular to the ribbed walls at various locations. They observed that strong secondary flow motion occurs over the entire duct crosssection for the inclined ribs. The flow structures between two consecutive ribs show that the fluid flows along the ribs from one end of the ribs to the other end, and then turns back at the transverse center.An experimental study was carried out by Cioncolini and Santini [2] in 2006 to investigate the transition from laminar to turbulent flow in helically coiled pipes. Coil diameter to tube 
diameter was from 6.9 to 369 , and twelve such coils have been tested. From direct observation of the experimental friction factor profiles the interaction between turbulence emergence and coil curvature has been analyzed. The experimental data compare favorably with existing results and reveal new features. The secondary flow in a curved duct of square cross-section is investigated experimentally by Yamamoto et. al. [3] in 2006.Three walls of the duct (except the outer wall) rotate around the center of curvature and an azimuthal pressure gradient was imposed. The variation of the flow patterns with change of flow parameters is compared with that of numerical calculations and was found to be in good agreement.A study on flow transition and development in circular and rectangular ducts was reported by Zanounet. al. [4] in 2009. The hot-wire anemometer was used to carry out measurements close to the circular duct exit; the Laser Doppler Anemometry (LDA) was utilized for the measurements. Transition criteria in both ducts were discussed, reflecting effects of flow geometry, entrance flow conditions, and entrance length on the transition Reynolds number. A laminar behavior was maintained up to $\mathrm{R}_{\mathrm{e}}=15.4 \times 10^{3}$ and $\mathrm{R}_{\mathrm{e}}=2 \times 10^{3}$ in the circular and rectangular ducts, respectively, and the transition was observed to take place at different downstream positions as the inlet flow velocity varied. Secondary flow in semicircular ducts is reported by Larsson ET. al. [5] in 2011. They found that the secondary flow in semicircular ducts consists of two pairs of counter rotating corner vortices, with a velocity in the range reported previously for related configurations. Agreement between simulation and experimental results are excellent when using a second moment closure turbulence model, and when taking the experimental and numerical uncertainty into account.

\section{EXPERIMENTAL SETUP}

Experiment is carried out using the facility of wind tunnel at the Aerodynamics Laboratory of National Institute of Technology, Durgapur. The experimental set up consists of a wind tunnel, which is driven by an electric motor of $5.5 \mathrm{~kW}$ power. The test piece is connected with the settling chamber via a constant area straight duct to ensure uniform velocity profile at the inlet section of the test piece. The geometry of the curved duct under test is shown in Fig. 1. It is a square $90^{\circ}$ curved duct of width $100 \mathrm{~mm}$ wide and $100 \mathrm{~mm}$ height with a centerline length of $750 \mathrm{~mm}$. It is constituted of four equal segments of $22.5 \square \square$ each. The entire test piece is made of Perspex sheet. Two straight constant area ducts of cross sectional area $100 \mathrm{~mm} \times 100 \mathrm{~mm}$ and $100 \mathrm{~mm}$ long are connected as extension pieces at the inlet and exit of the test piece. Middle points of all the six segments are considered as six sections and they are assigned as Inlet Section, Section - A, Section- B, Section - C, Section- D and Outlet Section. The wall static pressure is measured with the help of an inclined multi-tube manometer. At each face of the respective sections twelve locations are selected to determine the wall static pressure. A total of 288 locations on the test duct had been studied. Measuring locations of each selection points is shown in Fig.2.

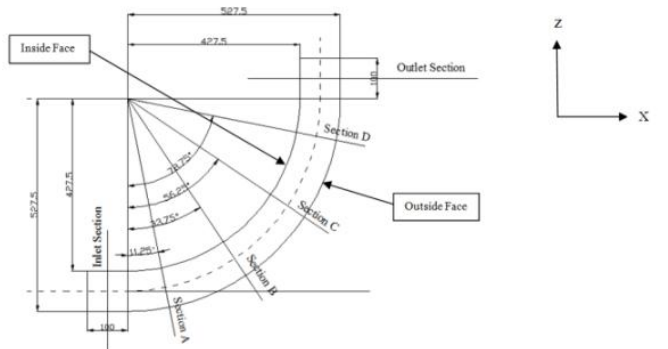

Fig. 1: Geometry of Curved Duct

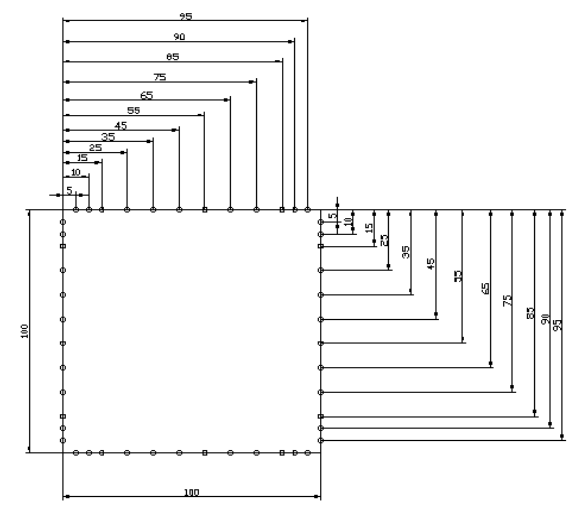

Fig. 2: Measuring locations of drill points

\section{RESULTS AND DISCUSSIONS}

Fig. 3.1(a), 3.1(b), 3.1(c) and 3.1(d) shows the distribution of wall static pressure for inlet average air velocity of $20 \mathrm{~m} / \mathrm{s}$. 3.1(a) clearly indicates a continuous decrease in normalized wall static pressure over the top surface along the direction of flow. The figure also depicts that the high pressure zone is built up near outside face which indicates the bulk movement of flow towards the inside face due to centrifugal action of the high velocity fluid. This is a probable indication of the development of secondary motion between the curved surfaces from the outside face to the inside face.Fig. 3.1(b) shows the normalized wall static pressure contours of the inside face. The contours show that high pressure zones accumulated near the top and bottom walls lead the movement of the flow from the top and bottom walls towards the mid plane.Fig. 3.1(c) shows 
normalized wall static pressure distribution over the bottom surface along the direction of flow which depicts the same type of observation as indicated in Fig. 3.1(a).Fig. 3.1(d) shows the normalized wall static pressure contours for the outside face where the existence of the high pressure zone may be noted at the mid section. This indicates the movement of flow from the mid plane towards the top and bottom surfaces signifying the possible development of counter rotating vortices along the flow passage.

Fig. 3.2(a), 3.2(b), 3.2(c) and 3.2(d) for inlet average air velocity of $40 \mathrm{~m} / \mathrm{s}$ and that of Fig. 3.3(a), 3.3(b), 3.3(c) and 3.3(d) for inlet average air velocity of $60 \mathrm{~m} / \mathrm{s}$ depict the exact same behavior as for their respective faces when studied for the inlet average velocity of $20 \mathrm{~m} / \mathrm{s}$.

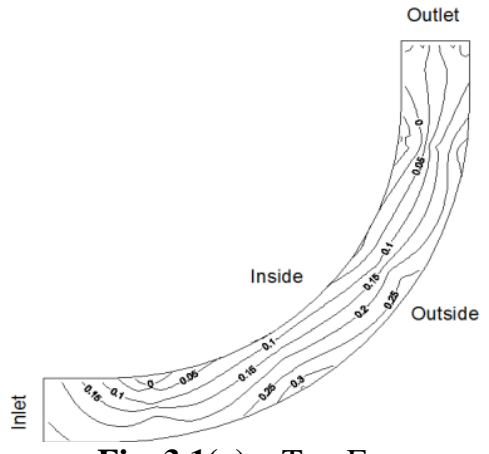

Fig. 3.1(a) - Top Face

Top

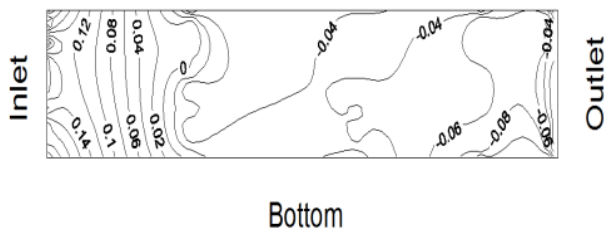

Fig. 3.1(b) - Inside face

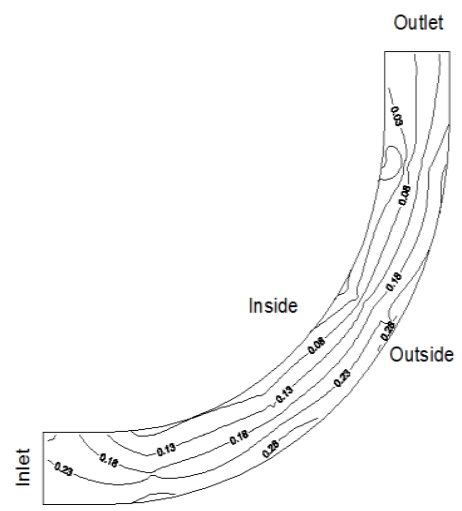

Fig. 3.1(c) - Bottom Face

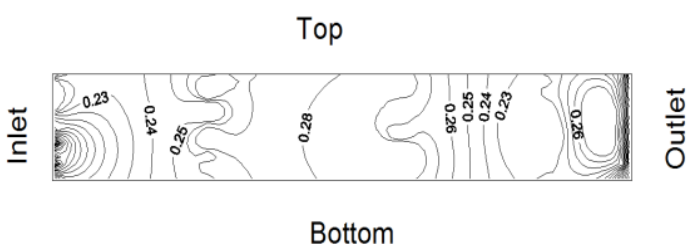

Fig. 3.1(d) - Outside Face

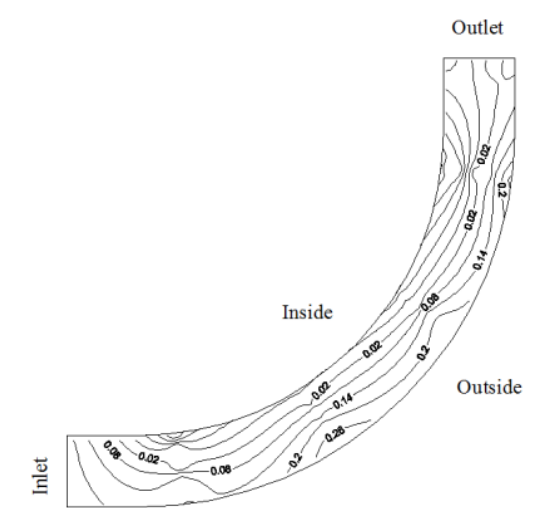

Fig. 3.2(a) - Top Face

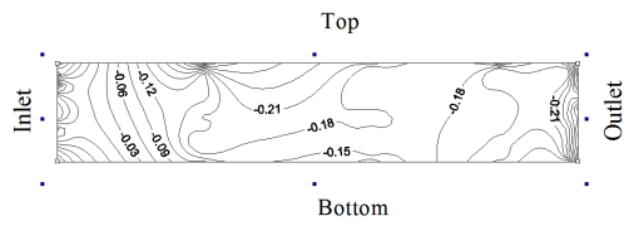

Fig. 3.2(b) - Inside Face

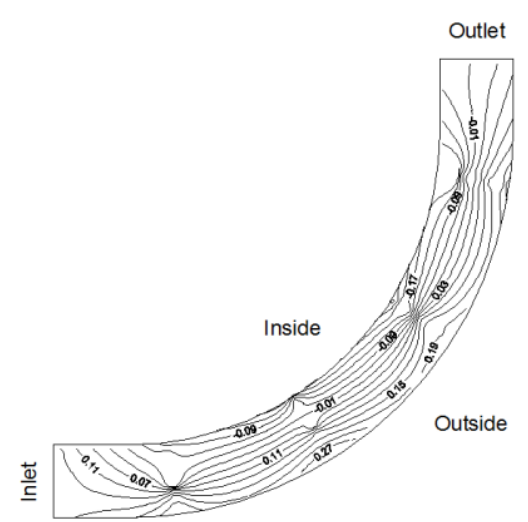

Fig. 3.2(c) - Bottom Face

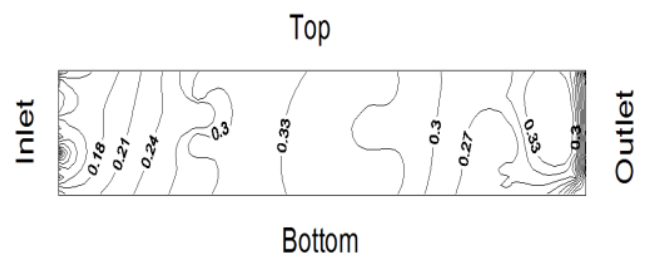

Fig. 3.2(d) - Outside Face 


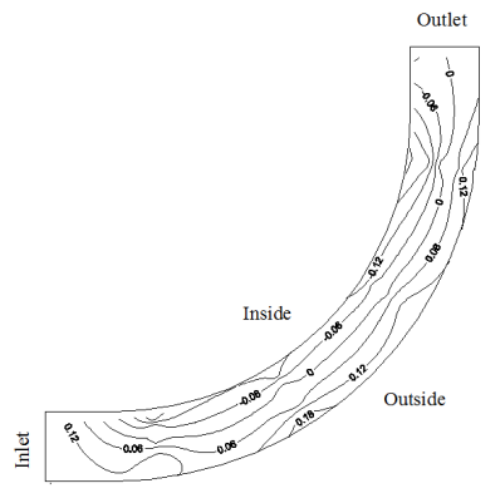

Fig. 3.3(a) - Top Face

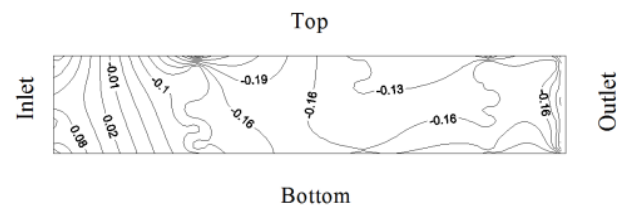

Fig. 3.3(b) - Inside Face

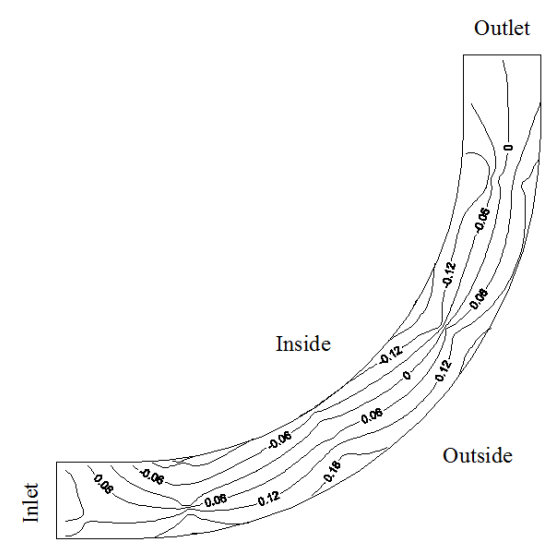

Fig. 3.3(c) - Bottom Face

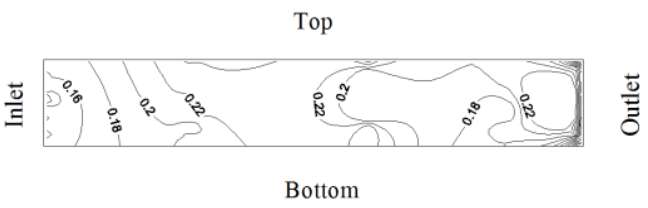

Fig. 3.3(d) - Outside Face

\section{CONCLUSIONS}

From the present investigation the following conclusions have been drawn:

a) We observe a continuous decrease of the normalized wall static pressure from the inlet to exit section of the curved duct for each of the top, bottom, inside and outside surfaces for the three velocities considered viz. $20 \mathrm{~m} / \mathrm{s}, 40 \mathrm{~m} / \mathrm{s}$ and 60 $\mathrm{m} / \mathrm{s}$. However, the outside face records a decrease which is comparatively lesser than other three faces. b) The minimum and maximum wall pressures have occurred at the inner and the outer wall respectively under the influence of the radius of curvature and angle of turn of the duct.

c) The bulk flow shifting from outer wall to the inner wall along the flow passage of curved duct is very instinct.

d) Flow at exit is purely non-uniform in nature due to the strong secondary motion.

e) Due to the imbalance of centrifugal force and radial pressure gradient, secondary motions in the form of counter rotating vortices have been generated within the curved duct. This may be termed as pressure driven secondary flow.

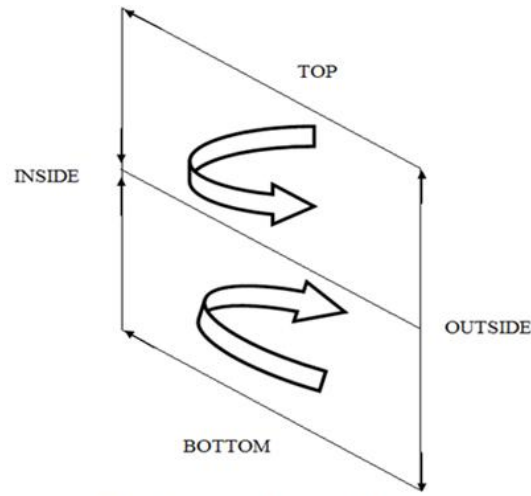

Fig: Counter Rotating Vortices

\section{REFERENCES}

[1] X. Gao and B. Sunden, "Effects on inclination angle of ribs on the flow behavior in rectangular ducts", Trans ASME, Journal of Fluid Engineering, Volume 126, 2004, pp. 692-699.

[2] Andria Cioncolini and Lorenzo Santini, "An experimental investigation regarding the laminar to turbulent flow transition in helically coiled pipes", Experimental Thermal and Fluid Science, 2006, Volume 30, pp. 380-397.

[3] Kyoji Yamamoto, XiaoyunWub, Kazuo Nozakib, Yasutaka Hayamizub, "Visualization of Taylor-Dean flow in a curved duct of square cross-section", Fluid Dynamics Research, Volume 38, Issue 1, January 2006, pp. 1-8.

[4] E.S. Zanoun, M. Kito and C. Egbers, "A study on flow transition and development in circular and rectangular ducts", ASME, Journal of Fluid Engineering, June 2009, Volume 131, Issue 6, 061204, 10 pages.

[5] I.A.S. Larsson, E.M. Lindmark, T.S. Lundström and G.J. Nathan, "Secondary flow in semi-circular ducts", ASME, Journal of Fluid Engineering, 2011, Volume 133, Issue 10, 101206. 